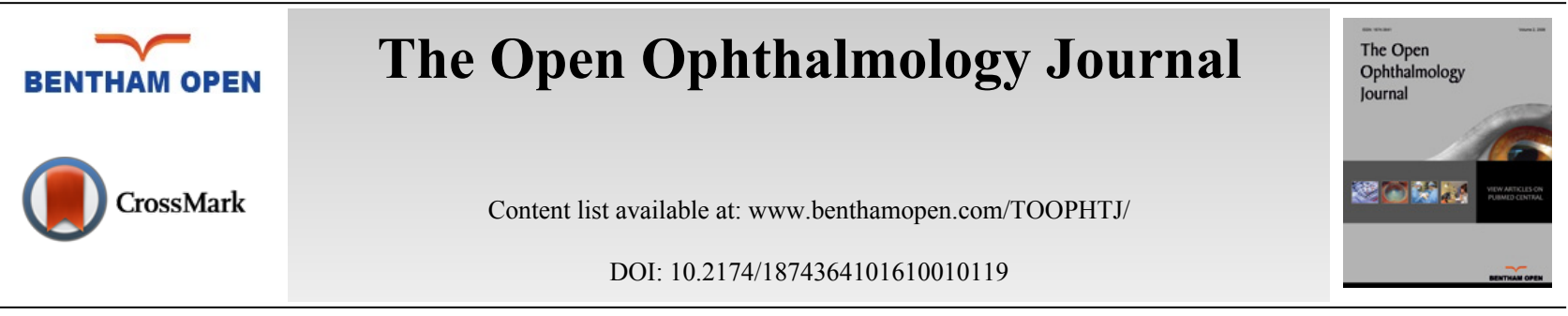

\title{
Evaluation of a Public Child Eye Health Tertiary Facility for Pediatric Cataract in Southern Nigeria I: Visual Acuity Outcome
}

\author{
Roseline E. Duke ${ }^{1, *}$, Adedayo Adio ${ }^{2}$, Sidney K. Oparah ${ }^{3}$, Friday Odey ${ }^{4}$ and Okon A. Eyo ${ }^{5}$ \\ ${ }^{I}$ Department of Ophthalmology, University of Calabar Teaching Hospital, Calabar, Cross River State, Nigeria \\ ${ }^{2}$ Department of Ophthalmology, University of Port Harcourt Teaching Hospital, Port Harcourt, Nigeria \\ ${ }^{3}$ Department of Medicine, University of Calabar Teaching Hospital, Calabar, Cross River State, Nigeria \\ ${ }^{4}$ Department of Pediatrics, University of Calabar Teaching Hospital, Calabar, Cross River State, Nigeria \\ ${ }^{5}$ Department of Community Medicine, University of Calabar Teaching Hospital, Calabar, Cross River State, Nigeria
}

Received: October 29, 2015

Revised: January 14, 2016

Accepted: January 16, 2016

\section{Abstract:}

\section{Purpose:}

A retrospective study of the outcome of congenital and developmental cataract surgery was conducted in a public child eye health tertiary facility in children $<16$ years of age in Southern Nigeria, as part of an evaluation.

\section{Materials and Method:}

Manual Small Incision Cataract Surgery with or without anterior vitrectomy was performed. The outcome measures were visual acuity (VA) and change (gain) in visual acuity. The age of the child at onset, duration of delay in presentation, ocular co-morbidity, non ocular co-morbidity, gender, and pre operative visual acuity were matched with postoperative visual acuity. A total of 66 children were studied for a period of six weeks following surgery.

\section{Results:}

Forty eight $(72.7 \%)$ children had bilateral congenital cataracts and $18(27.3 \%)$ children had bilateral developmental cataracts. There were $38(57.6 \%)$ males and $28(42.4 \%)$ females in the study. Thirty Five $(53 \%)$ children had good visual outcome (normal vision range $6 / 6 /-6 / 18)$ post-operatively. The number of children with blindness (vision <3/60) decreased from $61(92.4 \%)$ pre-operatively to $4(6.1 \%)$ post-operatively. Post operative complication occurred in $6.8 \%$ of cases six week after surgery. Delayed presentation had an inverse relationship with change (gain) in visual acuity $(r=-0.342 ; p$-value $=0.005)$. Pre-operative visual acuity had a positive relationship with post operative change (gain) in visual acuity $(r=0.618$; $p$-value $=0.000)$.

\section{Conclusion:}

Predictors of change in visual acuity in our study were; delayed presentation and pre-operative VA. Cataract surgery in children showed clinical benefit.

Keywords: Bilateral cataracts, Clinical benefit, Congenital, Developmental, Outcome, Southern Nigeria, Visual acuity.

\section{INTRODUCTION}

Pediatric cataract is the commonest cause of surgically treatable blindness and severe visual impairment in children in Nigeria, with up to $79 \%$ of lens related blindness and severe visual impairment occurring from un-operated bilateral congenital or developmental cataract [1]. The causes of blindness and severe visual impairment in children with

\footnotetext{
* Address correspondence to this author at the University of Calabar Teaching Hospital, Department of Ophthalmology, Pediatric Ophthalmology and Strabismus Unit, Calabar, Nigeria; E-mail: dr.roselineduke@gmail.com
} 
pediatric cataract ranges from the use of traditional 'couching' method with its associated complications, to the absence of appropriate surgical treatment, delayed presentation to hospitals and late surgical treatments. Amblyopia resulting from inadequate follow up after surgery, previous ocular surgeries and associated ocular co-morbidity also contribute to visual loss from cataract [1,2]. One of the Vision 2020 priorities in childhood blindness is the treatment of congenital cataract for which such children are to receive appropriate surgery, with immediate and effective optical correction, in suitably equipped specialist centers [3]. While it is important to establish community programs to facilitate adequately equipped early detection and presentation of children with congenital and developmental cataracts to eye care facilities [4], child eye care facilities which offer timely and appropriate surgical and optical treatment is key to successful treatment. With child eye care facilities in place offering pediatric cataract services, it becomes critical to evaluate the outcome of surgical intervention in such facilities and by surgeons. This is so as to monitor the effect of the surgical intervention and also to develop new ways to improve on the surgical outcomes.

There are various ways of evaluating the surgical outcome after surgery for congenital and developmental cataract. Such an evaluation can occur using various end points such as assessment of functional ability, pediatric quality of life assessment, educational attainment of the child and visual acuity measurements. In assessing the outcome of cataract surgery, visual acuity is traditionally utilized for interpretation, even though objective measures alone, such as visual acuity (VA) are recognized to be inadequate descriptors of functioning, and even less predictive of health-related quality of life (HRQOL) for pediatric cataract [5]. Nevertheless, Visual acuity still remains the easiest objective measure of the surgical intervention for cataract in children. Furthermore, it is used to determine the need for additional specific interventions after surgery. Various reports have proffered factors that predict and influence visual acuity outcome, to include age, time of intervention, morphology of cataract, surgical techniques including peculiar pediatric complications, choice of aphakic correction as well as the changing refraction and follow up care of the children [6, 7].

A public (federal government owned) child eye health facility in the Southern region of Nigeria was evaluated for its pediatric cataract system of care. This article reports part of the evaluation which is to assess the visual acuity outcome of congenital and developmental cataract surgery performed and to identify related factors pertaining to surgical outcome.

\section{MATERIAL AND METHODS}

A cross sectional retrospective chart review was performed for all patients who underwent pediatric cataract surgery between January 2011 and December 2012. Data for congenital and developmental cataracts were extracted. Congenital cataract was defined as cataract that developed within the first year of life or the signs of poor fixation signifying early onset. Developmental cataract was defined as a cataract that develops after the first year of life. Delayed presentation was defined as the time between detection by the caregiver for the first time and the time when the child was presented for surgical eye care in the University of Calabar Teaching Hospital (UCTH). The term 'visual acuity change' is defined as the gain resulting from the difference between the pre operative VA and the post operative VA of at least one Snellen acuity line or equivalent Snellen acuity line, This is also referred to as 'clinical benefit' in our study. The age at onset is defined as the time the career first noticed the eye problem. Data were collected on patient demographics, preoperative characteristics, intra operative complications, and post operative outcomes in the first six weeks. History taking and examination of the children were performed in the clinic and examination under anesthesia done prior to surgery. The morphology of the cataract was determined using slit-lamp bio-microscopy or the operation microscope after general anesthesia. Routine investigation of A \& B ocular ultrasound scan. Echo cardiogram was requested for all the patients prior to surgery by the anesthesiologist to assess for anesthesia fitness. The pediatricians and anesthesiologists examined and reviewed every child prior to surgery, certifying each child fit for surgery.

All patients had manual small incision cataract surgery (MSICS) performed. Bilateral simultaneous cataract surgery was carried out except for a few patients who had the second eye done after two days, due to anesthetic reasons. Patients below 4 years of age underwent primary capsulorhexis or vitreorhexis with or without anterior vitrectomy using : Portable Beaver Visitec, Waltham, MA or Alcon Phaco Laureate system- anterior vitrectomy. The utilization of the machines depended on cost, availability of a functioning anterior vitrectomy machine and the age of the patient. MSICS was performed in the following manner; first a scleral incision $1 \mathrm{~mm}$ from the limbus was performed, and a 1.5 $\mathrm{mm}$ scleral tunnel created; to dilate and maintain the dilated pupil, intra cameral adrenaline was used in the strength of $1: 10000$ of $0.3-0.5 \mathrm{mls}$ on entry into the anterior chamber. Anterior capsulorhexis or vitreorhexis was performed, in the event of a failure or the presence of a membranous cataract, intracapsular scissors was utilized. Cortical matter was washed out manually with a simcoe cannula and the nucleus was extracted using the vitrector or manually. Posterior 
capsulotomy and shallow anterior vitrectomy was performed routinely in children less than four years old. Fibrinous membranes that formed over the iris intra operatively were peeled off at the end of surgery. At least one stitch using 10.0 non-absorbable nylon suture was applied in all cases and the wound hydrated. The sutures were removed in most cases, at the next examination under anesthesia. The contact A scan technique for intraocular lens (IOL) power calculation using the IOL master to elicit the SRKII or HofferQ formulas was used. Twenty percent (20\%) and 10\% of the calculated power was deducted in patients 2-4-years-old and 5-7-years-old, respectively. We routinely aimed for hypermetropia except when we suspected poor caregiver compliance with spectacles. Acrylic hydrophobic preloaded single piece foldable intraocular lenses by Appassamy were implanted in children older than 3 years and older and older. The target location of the IOL was into the capsular bag. However, the information on the exact location at surgery of the intraocular lens on retrospective post-operative note review was lacking. All the children had surgery under general anesthesia with endotracheal intubation or the use of a laryngeal mask. Surgeries were performed by the same pediatric ophthalmic surgeon. Post operatively, all patients without IOLs were placed on topical atropine $0.5 \%$ or $1 \%$, and $1 \%$ mydriacyl for children that had IOLs. Topical steroids, dexamethasone and antibiotic ciprofloxacin were used for a minimum of 6 weeks. Children who did not have IOLs inserted were provided aphakic glasses on discharge from the hospital. The estimated power of the immediate spectacle was obtained based on the age of the child, the expected refractive power for age, aphakia, the axial length and the need for accommodation consequent upon surgery. The minimum number of days of hospital stay is four. Appropriate spectacle correction for children with or without IOLs was performed at the $6^{\text {th }}$ week of follow up. Best corrected visual acuity (BCVA) with spectacle correction was assessed with age appropriate acuity testing techniques, performed by an ophthalmic assistant or optometrist. Refraction was performed first objectively by the ophthalmologist during a comprehensive examination which included essentially a fundus examination using a $28 \mathrm{D}$ lens. This was followed by dispensing in preverbal or children or those with multiple disabilities. In verbal children objective retinoscope was followed by the use of the auto refractor/keratomater or immediately a subjective refraction by the optometrist was performed for distant and near vision. Examination under anesthesia was performed for a more thorough assessment in children who could not cooperate for a detailed eye examination in the clinic. Visual Acuity (VA) was assessed using recognition methods such as ability to fix and follow lights/objects, Central Sustained Maintained (CSM)6/9 - 6/6; CSNM -6/36 - 6/60, Unsteady central fixation < 6/60; hundred and thousand sweet test, as well as use of the Lea symbols, and the Snellen chart. The final VA outcome was selected as the visual acuity in the better eye at the last post-operative follow-up not later than the sixth week. Early complication was defined as complication seen within a period of six weeks after surgery.

Data were analyzed with SPSS version 16 (IBM Corp., New York, NY, USA). A p- value of less than 0.05 was considered statistically significant. In univariate analysis, for a continuous variable, independent sample t-test was used while for analysis of two categorical variables, a Chi-square test was used. Correlation was determined using Pearson correlates while for multivariate analysis linear regression was used. The research ethics committee of the University of Calabar Teaching Hospital Calabar, Cross River State approved this study.

\section{RESULTS}

One hundred and forty-six (146) children were scheduled to have cataract surgery during the period of study, of these, 103 (70\%) children had primary surgery performed. The remaining 43(29.4\%) did not present for surgery during the scheduled period. However, some of the families still brought their children for surgery at a later time. A total of 87 of the $103(84.5 \%)$ children had bilateral congenital and developmental cataract, while the remaining $16(15.5 \%)$ had traumatic cataract and unilateral cataract. Of the 87 children with congenital and developmental cataract, $71(81.6 \%)$ case notes were retrieved for data extraction. The remaining $17(18.3 \%)$ case notes were missing. Of the $71(81.6 \%)$ entries made, data were incomplete in the column of visual acuity for distance and near. These were excluded from the analysis, leaving $66(75.8 \%)$ only bilateral cataracts with,accurate and detailed data entries. Patients came for surgery from two main geopolitical zones of Nigeria, namely the South South (SS) 46(69.7\%) and South East(SE) 20(30.3\%). Data from 66 children were accordingly analyzed, $48(72.7 \%)$ children had bilateral congenital cataract and $18(27.3 \%)$ children had bilateral developmental cataract. There were 38(57.6\%) males and 28 (42.4\%) females (see Table 1).

There was no difference in the outcome of VA between the males and females. Fifty nine (89.3\%) children presented for at least one follow up examination during the six weeks period. Table 2 shows the proportion of children with visual acuity categorization preoperative and post operatively. Good visual outcome (normal vision range 6/6/ $-6 / 18)$ post operatively was seen in $35(53 \%)$ of the children. Table 3 shows the frequency of each ocular co morbid condition. A total of $45(68.2 \%)$ children had coexisting ocular co morbidity, while Table 4 shows the relationship between ocular morbidity and post-operative change (gain) in visual acuity. 
Table 1. Gender distribution and means.

\begin{tabular}{|c|c|c|c|c|}
\hline Parameter & Male & Female & Overall & p-value \\
\hline Number & $38(57.6 \%)$ & $28(42.4 \%)$ & $66(100 \%)$ & \\
\hline Mean age (in years) \pm Std Dev & $4.5 \pm 2.9$ & $5.7 \pm 4.3$ & $5.0 \pm 3.6$ & 0.157 \\
\hline Age range (in years) & $0.3-11$ & $0.3-15$ & $0.3-15$ & \\
\hline Mean age at onset (in years) \pm Std Dev & $2.9 \pm 2.6$ & $4.7 \pm 4.2$ & $3.6 \pm 3.4$ & 0.041 \\
\hline Range of age at onset (in years) & $0.2-9.0$ & $0.2-15$ & $0.2-15$ & \\
\hline Mean duration of delayed presentation (in years) \pm Std Dev & $2.6 \pm 2.1$ & $2.8 \pm 3.4$ & $2.7 \pm 2.7$ & 0.739 \\
\hline Range of duration of delayed presentation (in years) & $0.3-8.0$ & $0.2-15$ & $0.2-15$ & \\
\hline
\end{tabular}

N/B: Std Dev = Standard deviation

Table 2. Proportion of children with visual acuity grading before and after surgery.

\begin{tabular}{|c|c|c|c|}
\hline VA category & Grade of vision & Pre-operative (\%) & Final VA (\%) \\
\hline $6 / 6-6 / 18$ & Normal & $2(3.0 \%)$ & $35(53 \%)$ \\
\hline$<6 / 18-6 / 60$ & Moderate visual impairment & $1(3.0 \%)$ & $27(40.9 \%)$ \\
\hline$<6 / 60-3 / 60$ & Severe visual impairment & $61(92.4 \%)$ & $4(6.1 \%)$ \\
\hline$<3 / 60-$ PL & blindness & $66(100 \%)$ & $66(100 \%)$ \\
\hline Total & & & 6 \\
\hline
\end{tabular}

Table 3. Frequency and distribution of each coexisting ocular co morbidities with cataract.

\begin{tabular}{|c|c|c|}
\hline Type of co-morbid eye disease & Frequency & Percentage \\
\hline Nystagmus & 35 & 24 \\
\hline Micro-ophthalmous & 21.0 & 36.4 \\
\hline Strabismus & 31.8 & 4 \\
\hline Glaucoma/Buphthalmous & 6.1 & \\
\hline
\end{tabular}

Table 4. Ocular co morbidity and change (gain) in VA.

\begin{tabular}{|c|c|c|c|}
\hline Parameter & $\begin{array}{c}\text { No ocular co-morbidity } \\
(\mathbf{n}=\mathbf{2 1})\end{array}$ & $\begin{array}{c}\text { Ocular co-morbidity } \\
(\mathbf{n}=\mathbf{4 5})\end{array}$ \\
\hline Mean VA change & 1.4357 & 1.1633 \\
\hline Standard deviation & \pm 0.44865 & 0.59423 \\
\hline Range of VA change & $0.0-2.0$ & $0.0-2500$ \\
\hline
\end{tabular}

$\mathrm{t}=2.063 ; \mathrm{p}=0.044 ; 95 \%$ C.I $=-0.0073$ to 0.5375 .

Thirty two (48.5\%) children presented with non ocular co-morbidity which included, cardiac disease, delayed milestones, mental retardation, deafness, epilepsy and physical handicap. However, there was no significant reduction in visual acuity seen in children with non ocular co-morbidity, including children with congenital rubella syndrome [8]. Thirty three $(65.2 \%)$ children had intraocular lens implantation. There was no significant change in VA between children who had IOL implanted and those who did not $(\mathrm{t}=0.6$; $\mathrm{p}$-value $=0.619)$.

Intra operative complications were reported in our study per eye of which, failed capsulorhexis was seen in $35(26.5 \%)$ eyes where manual capsulorhexis was attempted, the alternative in such cases was to perform a vitreo-rhexis. Post-operative complications were seen in 9 out of 132 eyes $(6.8 \%) ; 2(1.5 \%)$ had peaked pupils and $3(2.3 \%)$ had corneal opacity at the keratome entry site. Two (1.5\%) had IOL decentration, 2 (1.5\%) patients had a severe fibrinoid reaction. The change (gain) in VA, derived by subtracting the pre-operative VA from the final post-operative value, had a negative relationship with the duration of delayed presentation $(\mathrm{r}=-0.342$; $\mathrm{p}$-value $=0.005)$, age at onset $(\mathrm{r}=-0.253$; $\mathrm{p}$-value $=0.040)$ and the age at surgery $(\mathrm{r}=-0.297$; $\mathrm{p}$-value $=0.016)$, respectively. On the other hand, there was a positive relationship between change (gain) in VA and the pre-operative VA $(r=0.618$; $p$-value $=0.000)$. There was no difference in the duration of delayed presentation between those who had congenital and developmental cataract ( $p$ value $=0.183$ ) or between males and females (see Table 1).

Pre-operative visual acuity $(\beta$ coefficient $=0.604 ; p$-value $=0.000)$ and duration of delayed presentation $(\beta$ 
coefficient $=-0.315 ; \mathrm{p}$-value $=0.010)$ were independent predictors of the post-operative change (gain) in visual acuity.

\section{DISCUSSION}

The concept of a Child Eye Health Tertiary Facility (CEHTF) was conceived to serve a defined population of 10 million in developing countries [4]. Cataract surgery is one of the commonest procedures performed in the facility. It is also an important step in the restoration of vision and function lost from pediatric cataract, but proper follow-up with optical correction and amblyopia treatment make it more effective.

Similar to other studies in the same region, males presented more than females to take up eye surgery.[9] This is probably for the reason of a higher societal value for the male child. However, the outcome of VA did not differ between the genders. Even though $62 \%$ of children achieved a VA of at least $6 / 60$, similar to what was seen in another study in Nigeria and reported by Cogan et al. [2,9], the target acceptable good visual outcome of $95 \%$ in the 6/6-6/18 visual acuity category was not attained. The reasons for the poor visual acuity outcome seen in this group of children may be attributed to amblyopia as seen in the mean age of presentation as well as the duration of delayed presentation, which exceeded the critical period of visual development evident by the presence of nystagmus. This is similar to studies in Tanzania and India where occlusion amblyopia was responsible for up to $80 \%$ reduction in visual acuity [10, 11]. Also responsible for the poor estimate of visual acuity outcome, may be the inaccuracy of visual acuity taken in the children, due to a busy clinic or inadequate time spent to perform and obtain an accurate measure. It was noticed that some children did not have visual acuity documented and such children were not included in the study. A study reported $59.5 \%$ of preoperative visual acuity to be commonly recorded by the fixation pattern and $29 \%$ post-operative use of the method [12]. Investigation into the methods and the use of protocol in performing children's VA assessment is recommended.

Intraocular lens implantation prevents amblyopia in children who have had cataract surgery mainly because of the provision of a constant visual stimulation to the brain. Probably, because of the short duration of assessment of outcome in this study the effect of IOL on visual outcome was not significant, even though long term reports from India has shown that visual acuity gets better with time [12]. However, newer IOL power calculations and methods which give better visual acuity for children are available.

The proportion of children with immediate post operative complication was low and less fibrinoid reactions were seen in our study. This is different from other studies where an intense fibrinoid reaction was seen during the early postoperative period [9]. Care was taken to peel of any fibrin layer over the anterior iris surface with minimal release of iris pigments at the end of surgery. Furthermore, this difference with other studies, may be because of the time expected for this reaction to occur against the time of discharge, the use of cycloplegic agents and especially intense topical steroid with a frequency of every 30 minutes in the first three days at least. This was instilled by a parent or care giver after training on the method of eye drop instillation. Automated irrigation and aspiration method may reduce the presence of microorganism which could incite an inflammation in cases such as rubella cataracts.

Further complications of a peaked pupil most likely from inadequate vitreous clearance are of concern as one considers the incidence of late onset retinal detachment as documented in some studies. Manual surgical procedures similar to Extracapsular Cataract Extraction and even Manual Small Incision Cataract Surgery with or without IOL implantation in pediatric eyes is associated with various problems and can lead to early complications as seen in our study or late permanent visual disability, as reported in another study [13]. Moreover, phacoemulsification in older children has been seen to give a better outcome in the long run for paediatric cataracts [13]. Recent surgical techniques using an endoilluminator to ensure guided, adequate and complete mechanized anterior vitrectomy has been suggested and the technique is recommended [14]. The use of preservative free intracameral dexamethasone of $0.4 \mathrm{mg} / 0.1 \mathrm{ml}$ is discouraged in our center because the long term effects of their use in children is not clear enough, especially in view of the development of aphakic glaucoma which is difficult to treat in our setting [14]. Though its use is beneficial in enabling visualization of the vitreous to indicate the completion of anterior vitrectomy, reducing intraocular inflammation as well as protecting against anterior membrane formation.

We have not reported any case of early endophthalmitis even though we did not use intracameral antibiotics as this would add to the cost of surgery to the patients. More studies in our environment regarding the use of prophylactic intracameral antibiotics needs to be investigated. Early posterior capsular opacity was not seen because of the primary capsulotomy performed essentially and an anterior vitrectomy, but most especially because we are not reporting intermediate and late complications of cataract surgery in children. 
Clinical benefit and gain from surgery was evident in our study. It is interesting to note that with regards to the visual acuity, the clinical benefit 'endpoint for congenital and developmental cataract surgery may not be the traditional 'good outcome' of better than 6/18 visual acuity measurement but an immediate improvement in visual acuity enough to begin the path of visual stimulation and vision development. In our study, a strongly positive significant correlation between pre operative and post operative visual acuity was seen similar to other studies $[6,15]$.

Some authors have suggested that children with bilateral symmetrical cataract should have unilateral surgery performed per surgical session, especially when IOL is implanted as this is a better protocol for surgery since it affords the opportunity to reassess intraocular lens power implant. It is postulated that the outcome for the second eye would be better since it gives the opportunity for a better optical correction [16]. In our peculiar circumstances, given manpower and numerous logistic challenges especially with anesthesia, it is quite difficult to offer surgical service twice to each patient. Therefore performing bilateral simultaneous surgery at one setting is the norm unless adequate anesthesia is unavailable for the two eyes to be operated on during one surgical session. Furthermore, our study focused on children with bilateral cataract who have a better outcome and a better prognosis than unilateral cataract surgery [17].

The presence of ocular co morbidity has been associated with poorer outcomes [11]. A similar situation is reported in our study. The presence of ocular co morbidity alerts on the visual acuity expectation in the child as well as the expected complication at and following surgery. This information aids in counseling families on outcome expectation.

It was noted that children with clinical congenital rubella did not have significantly probably poorer visual outcome. Non ocular co-morbidity did not significantly influence visual acuity outcome but may influence the response perception, and cooperation during visual acuity assessment. Coexisting non ocular co morbid conditions may be more appropriate in reflecting the extent to which systemic conditions influences the interpretation, utilization of vision and health related quality of life rather than on visual acuity outcome.

While age at onset of the disease is important in influencing post operative VA, the delayed presentation time, is more important as seen in our study and other studies [9, 18, 19]. The independent predictors of a change in visual acuity in our study were mainly delayed presentation (inverse relationship) and pre-operative VA. Gogate et al documented a positive direct association between visual outcome and follow up visit [19]. A proportion of patients attended only one follow up visit. The reasons for this needs to be investigated in this environment.

The study has two major limitations: Retrospective retrieval of data and loss to follow-up.

Different techniques were used for measuring visual acuity so internal validity of visual acuity assessment is low.

\section{CONCLUSION}

The expectation of good visual outcome from pediatric cataract surgical intervention, by skilled ophthalmic surgeons, families, children and investors is increasing. Therefore, continued efforts to improve on the surgical techniques specific to pediatric cataract care are critical. Newer improved surgical techniques in the management of pediatric cataract especially involving automated instrumentation and options for IOL calculation are available to the community. Investigation into the pediatric cataract system of care from the community to the CEHTF and back to the community, including assessing the use of clinical protocols in this CEHTF would be complimentary to this study in giving a comprehensive evaluation report.

\section{CONFLICT OF INTEREST}

The authors confirm that this article content has no conflict of interest.

\section{ACKNOWLEDGEMENTS}

Declared none.

\section{REFERENCES}

[1] Duke R, Otong E, Iso M, et al. Using key informants to estimate prevalence of severe visual impairment and blindness in children in Cross River State, Nigeria. J AAPOS 2013; 17(4): 381-4.

[http://dx.doi.org/10.1016/j.jaapos.2013.05.004] [PMID: 23911130]

[2] Congdon NG, Ruiz S, Suzuki M, Herrera V. Determinants of pediatric cataract program outcomes and follow-up in a large series in Mexico. J Cataract Refract Surg 2007; 33(10): 1775-80.

[http://dx.doi.org/10.1016/j.jcrs.2007.06.025] [PMID: 17889776] 
[3] Gilbert C, Foster A. Childhood blindness in the context of VISION 2020--the right to sight. Bull World Health Organ 2001; 79(3): 227-32. [PMID: 11285667]

[4] Agarwal PK, Bowman R, Courtright P. Child Eye Health Tertiary Facilities in Africa. J AAPOS 2010; 14(3): 263-6. [http://dx.doi.org/10.1016/j.jaapos.2010.02.007] [PMID: 20603061]

[5] Chak M, Rahi JS. The health-related quality of life of children with congenital cataract: findings of the British Congenital Cataract Study. Br J Ophthalmol 2007; 91(7): 922-6.

[http://dx.doi.org/10.1136/bjo.2006.109603] [PMID: 17244652]

[6] Gogate P, Khandekar R, Shrishrimal M, et al. Delayed presentation of cataracts in children: are they worth operating upon? Ophthalmic Epidemiol 2010; 17(1): 25-33. [http://dx.doi.org/10.3109/09286580903450338] [PMID: 20100097]

[7] Vasavada AR, Nihalani BR. Pediatric cataract surgery. Curr Opin Ophthalmol 2006; 17(1): 54-61. [PMID: 16436925]

[8] Duke R, Oparah S, Adio A, Eyo O, Odey F. Systemic Comorbidity in Children with Cataracts in Nigeria: Advocacy for Rubella Immunization. J Ophthalmol 2015 [Cited 2015 October 28];4. ID 927840 [Internet] Available from: http://www.hindawi.com/12972584/

[9] Umar MM, Abubakar A, Achi I, Alhassan MB, Hassan A. Pediatric cataract surgery in National Eye Centre Kaduna, Nigeria: outcome and challenges. Middle East Afr J Ophthalmol 2015; 22(1): 92-6. [http://dx.doi.org/10.4103/0974-9233.148356] [PMID: 25624681]

[10] Bowman RJ, Kabiru J, Negretti G, Wood ML. Outcomes of bilateral cataract surgery in Tanzanian children. Ophthalmology 2007; 114(12): 2287-92. [http://dx.doi.org/10.1016/j.ophtha.2007.01.030] [PMID: 17448539]

[11] Khanna RC, Foster A, Krishnaiah S, Mehta MK, Gogate PM. Visual outcomes of bilateral congenital and developmental cataracts in young children in south India and causes of poor outcome. Indian J Ophthalmol 2013; 61(2): 65-70. [http://dx.doi.org/10.4103/0301-4738.107194] [PMID: 23412523]

[12] Gogate PM, Sahasrabudhe M, Shah M, et al. Long term outcomes of bilateral congenital and developmental cataracts operated in Maharashtra, India. Miraj pediatric cataract study III. Indian J Ophthalmol 2014; 62(2): 186-95. [http://dx.doi.org/10.4103/0301-4738.128630] [PMID: 24618489]

[13] Matalia J, Anaspure H, Shetty BK, Matalia H. Intraoperative usefulness and postoperative results of the endoilluminator for performing primary posterior capsulectomy and anterior vitrectomy during pediatric cataract surgery. Eye (Lond) 2014; 28(8): $1008-13$. [http://dx.doi.org/10.1038/eye.2014.136] [PMID: 24946844]

[14] Gungor SG, Bulam B, Akman A, Colak M. Comparison of intracameral dexamethasone and intracameral triamcinolone acetonide injection at the end of phacoemulsification surgery. Indian J Ophthalmol 2014; 62(8): 861-4. [http://dx.doi.org/10.4103/0301-4738.141045] [PMID: 25230962]

[15] Krummenauer F, Landwehr I. Incremental cost effectiveness evaluation in clinical research. Eur J Med Res 2005; 10(1): 18-22. [PMID: 15737949]

[16] Al Shamrani M, Al Turkmani S. Update of intraocular lens implantation in children. Saudi J Ophthalmol 2012; 26(3): 271-5. [http://dx.doi.org/10.1016/j.sjopt.2012.05.005] [PMID: 23961005]

[17] Hennig A, Schroeder B, Gilbert C. Bilateral pediatric cataract surgery: outcomes of 390 children from Nepal and Northern India. J Pediatr Ophthalmol Strabismus 2013; 50(5): 312-9.

[http://dx.doi.org/10.3928/01913913-20130402-01] [PMID: 23565713]

[18] Gogate P, Patil S, Kulkarni A, et al. Barriers to follow-up for pediatric cataract surgery in Maharashtra, India: how regular follow-up is important for good outcome. The Miraj Pediatric Cataract Study II. Indian J Ophthalmol 2014; 62(3): 327-32. [http://dx.doi.org/10.4103/0301-4738.116465] [PMID: 24008794]

[19] Mwende J, Bronsard A, Mosha M, Bowman R, Geneau R, Courtright P. Delay in presentation to hospital for surgery for congenital and developmental cataract in Tanzania. Br J Ophthalmol 2005; 89(11): 1478-82. [http://dx.doi.org/10.1136/bjo.2005.074146] [PMID: 16234457]

(C) Duke et al.; Licensee Bentham Open.

This is an open access article licensed under the terms of the Creative Commons Attribution-Non-Commercial 4.0 International Public License (CC BY-NC 4.0) (https://creativecommons.org/licenses/by-nc/4.0/legalcode), which permits unrestricted, non-commercial use, distribution and reproduction in any medium, provided the work is properly cited. 